\title{
Cell sorting using efficient light shaping approaches.
}

\author{
Banas, Andrew; Palima, Darwin; Villangca, Mark Jayson; Glückstad, Jesper
}

\section{Published in:}

Proceedings of SPIE

Link to article, DOI:

$10.1117 / 12.2214165$

Publication date:

2016

Document Version

Publisher's PDF, also known as Version of record

Link back to DTU Orbit

\section{Citation (APA):}

Banas, A., Palima, D., Villangca, M. J., \& Glückstad, J. (2016). Cell sorting using efficient light shaping approaches. In J. Glückstad, D. L. Andrews, \& E. J. Galvez (Eds.), Proceedings of SPIE (Vol. 6764). [97640F-1] SPIE - International Society for Optical Engineering. Proceedings of SPIE - The International Society for Optical Engineering https://doi.org/10.1117/12.2214165

\section{General rights}

Copyright and moral rights for the publications made accessible in the public portal are retained by the authors and/or other copyright owners and it is a condition of accessing publications that users recognise and abide by the legal requirements associated with these rights.

- Users may download and print one copy of any publication from the public portal for the purpose of private study or research.

- You may not further distribute the material or use it for any profit-making activity or commercial gain

- You may freely distribute the URL identifying the publication in the public portal 


\author{
Cell sorting using efficient light shaping approaches \\ Andrew Bañas ${ }^{2}$, Darwin Palima ${ }^{1}$, Mark Villangca ${ }^{1}$, and Jesper Glückstad ${ }^{1,2}$ \\ ${ }^{1}$ Department of Photonics Engineering, Technical University of Denmark \\ Oersted Plads 343, DK-2800 Kgs. Lyngby, Denmark \\ ${ }^{2}$ OptoRobotix ApS, Scion DTU, Diplomvej 381, DK-2800 Kgs. Lyngby, Denmark \\ Email:amb@optorobotix.com \\ www.ppo.dk www.OptoRobotix.com www.GPCphotonics.com
}

\begin{abstract}
Early detection of diseases can save lives. Hence, there is emphasis in sorting rare disease-indicating cells within small dilute quantities such as in the confines of lab-on-a-chip devices. In our work, we use optical forces to isolate red blood cells detected by machine vision ${ }^{1}$. This approach is gentler, less invasive and more economical compared to conventional FACS systems. As cells are less responsive to plastic or glass beads commonly used in the optical manipulation literature, and since laser safety would be an issue in clinical use, we develop efficient approaches in utilizing lasers and light modulation devices. The Generalized Phase Contrast (GPC) method that can be used for efficiently illuminating spatial light modulators or creating well-defined contiguous optical traps is supplemented by diffractive techniques capable of integrating the available light and creating 2D or 3D beam distributions aimed at the positions of the detected cells. Furthermore, the beam shaping freedom provided by GPC can allow optimizations in the beam's propagation and its interaction with the catapulted cells.
\end{abstract}

Keywords: cell sorting, beam shaping, machine vision, GPC

\title{
1. INTRODUCTION
}

Cell sorting is important in cellular biology, health and medical research ${ }^{1,2}$. For example, the identification of rare diseases and subsequent isolation of infected cells among millions of others can be used to further study such disease. The early identification of diseases through the detection of rare circulating tumor cells, or rare anemia in the blood can be used to take preventive measures before the condition worsens ${ }^{3}$.

Given its well understood importance, devices for studying cells are being developed as early as the $60 \mathrm{~s}^{4}$. For example, one established method for cell sorting is based on flow cytometry or fluorescence-activated cell sorting systems (FACS). Flow cytometry works by queuing cells in a single line so they can be analyzed one by one. An alternative based on optical fractionation ${ }^{5}$ was also proposed for sorting. Sorting is possible as particles with different properties have characteristic deflections as they flow through an optical lattice. As particle motion in fractionation is not actively controlled, careful calibration of the optical lattice is required.

We take a different approach on sorting by using optical scattering forces as the sorting mechanism ${ }^{6}$ and machine vision to automate detecting particles of interest. Once detected, cells are directly pushed into another stream for later collection and study. Figure 1 illustrates our cell sorting approach. This approach may be compared with quality control of fruits as they are picked from a conveyor, in which case, machine vision is also employed. Machine vision had been successfully used to identify objects and automate tasks as early as the $80 \mathrm{~s}$ wherein it is applied in sorting in quality control, automated manufacturing, unmanned vehicles among many others. Until today, as processing hardware and image recognition algorithms continue to improve, and with the increasing amount of calibration image data, machine vision is actively used in diverse fields such as gaming and computing, unmanned vehicles, and person identification. Applied to optical manipulation, machine vision can be used to locate cells that deviate from normal healthy ones and then activate traps based on the cell's position enabling automated manipulation ${ }^{7}$ or dynamic stabilization ${ }^{8}$.

The Bio-Optofluidic Cell Sorter ${ }^{9}$ (cell-BOCS) is therefore designed to utilize machine vision to search for abnormalities in cells. Visible features on cell samples such as morphology, size or color are used as a basis of discrimination. As fluorescence is optional, samples can be prepared on solutions that keep them viable. The cell-BOCS's gentle sorting 
approach also preserves the cells viability. Keeping the cells alive are important for later studies as they can be increase in numbers through culturing and their reactions to possible treatment can be observed.

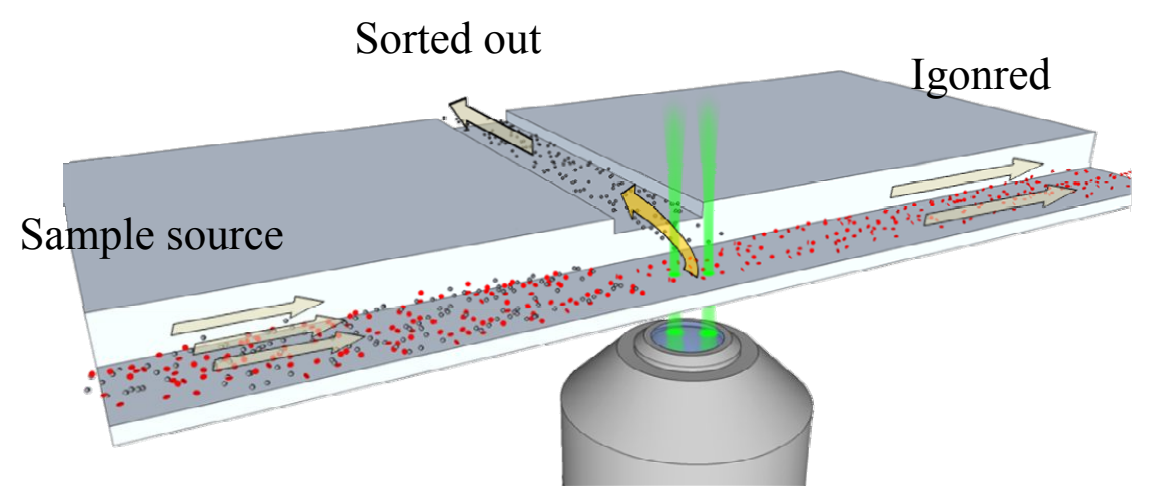

Figure 1. Sorting principle of the cell-BOCS. A sample mixture in a laminar flow is goes through a detection window that is viewed through the microscope. Cells that fit a certain criterion are located by processing the images grabbed through the microscope. Catapult traps are activated at the locations of these identified cells to push them to another flow channel where they are sorted out.

\subsection{Efficient laser utilization}

The cell-BOCS borrows features from our previously reported BioPhotonics Workstations ${ }^{10}$ (BWS) and both are actively being miniaturized ${ }^{9}$. Unlike the BWS, however, there is a higher incentive for a compact implementation in the cellBOCS for deployment in "non-optics" bio-oriented facilities. Efficient laser shaping would therefore allow much smaller lasers, reducing the footprint of the cell-BOCS. Other advantages are the reduced bill of materials, and the reduced risk of laser related injuries. Due to the expected scarcity of disease carrying cells, it would be typical to run the cell sorter for prolonged periods (hours to days), hence, a reduced operating power consumption would also be beneficial.

\section{LIGHT SHAPING STRATEGIES}

Although, microscopic phenomena can be altered through macroscopic ways, like introducing chemicals into sample medium, or controlling temperature, there are many instances wherein it is preferable to have localized and spatially controlled interactions. This is particularly true with cell sorting wherein a high concentration of the cells of interest is preferred. Light's ability to be shaped then be sent to microscopic environments, even sealed ones, like through the channels of a microfluidic chip, offers a very convenient way of exerting spatially distributed forces on biological samples. We will therefore consider some efficient and practical light shaping techniques that can be applied to cell sorting. This would include Generalized Phase Contrast, matched filtering Generalized Phase Contrast, and the use of binary diffraction gratings.

There are some common features in the light shaping techniques to be considered. First, in order to be efficient, the techniques should be classified as "phase-only modulation". This means that no light is blocked or deflected away in a subtractive manner in order to sculpt out the desired light pattern, as in amplitude modulation. Another common feature is the use of programmable phase-only spatial light modulators (SLM). Like video screens, SLM's are pixel addressed device. However, instead of controlling color transmittance, phase SLM pixels control the phase of light that reflects of each pixel. Hence, SLM's are convenient for applying phase transmittance functions to initially flat-phased light sources. Further details and considerations about the light shaping techniques will be discussed in the succeeding subsections.

\subsection{Generalized Phase Contrast}

Generalized Phase Contrast ${ }^{11}$ (GPC) is an extension to Zernike's phase contrast microscopy primarily designed for beam shaping ${ }^{11}$, but also finds used in quantitative phase imaging ${ }^{12,13}$ and optical encryption ${ }^{14}$. Phase modulated input light is focused through a static phase element, i.e. a phase contrast filter (PCF) and subsequently imaged in the far field or another Fourier lens. Hence, in the GPC method, an input phase pattern is directly mapped into an intensity pattern through the $4 f$ filtering configuration. This simple one-to-one pixel mapping lessens the computational requirements, 
enabling real-time reconfigurability as no iterative Fourier transform calculations are required. With the use of a $4 f$ imaging configuration, potentially disturbing light that would have otherwise been spent on the zero-order in a $2 f \mathrm{CGH}$ setup is utilized as a synthetic reference wave for forming patterns at the output via self-interference. Furthermore, since the output has a flat phase profile, GPC becomes convenient for certain volume oriented applications such as counter propagating optical traps mainly used in the BWS ${ }^{10,15}$ or catapulting detected cells at a height of $\sim 100 \mu \mathrm{m}$ in order to push it to another flow channel.

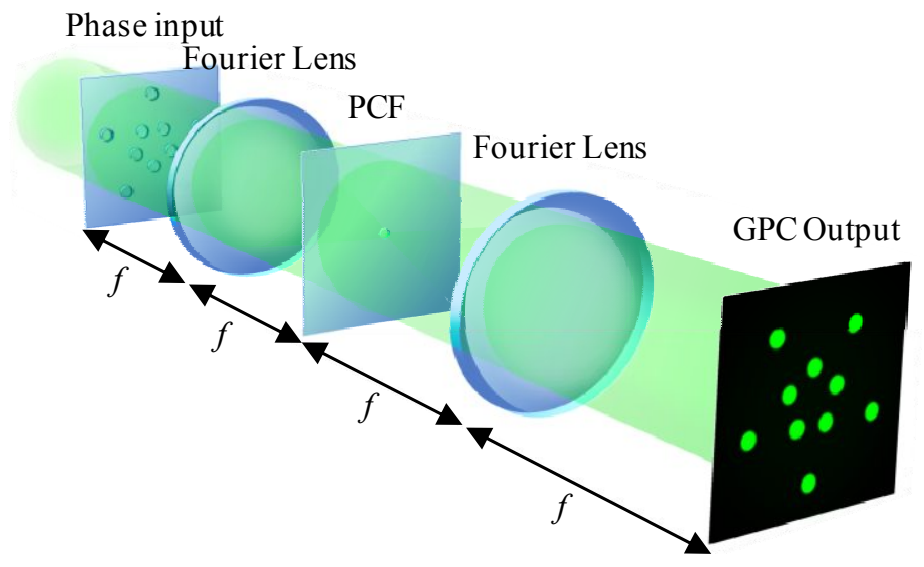

Figure 2. A Generalized Phase Contrast setup. Phase input patterns are mapped into output intensity patterns via common path interferometry. The PCF located at the Fourier plane shifts the input's lower frequencies that in turn serve as a reference wave, imaging the input phase pattern via interference.

GPC's $4 f$ mapping scheme also allows binary SLMs to be used without dealing with mirrored "ghost" copies of the pattern. This would be a problem with holography where the mirrored "twins" at higher orders will take up as much energy as the utilized off-axis patterns. Although GPC allows fast SLM addressing, its one-to-one pixel mapping sets an upper limit to how intense the output beam could be. GPC output would typically be around 4 times more intense than the input level, corresponding to constructive interference of the foreground pattern with the synthetic reference wave ${ }^{11}$. GPC's peak power, however, can be adjusted by choice of focal length of the final objective lens, at the expense of the size of field of view.

For Gaussian incident beams, GPC can be up to $84 \%$ efficient and the intensity gain can be up to $3 x^{16}$. However, unless using multiple smaller beams, there is a limited working area when using Gaussian illuminated GPC. With its use with static GPC Light Shapers ${ }^{17}$, Gaussian GPC can boost the illumination intensity on spatial light modulators, allowing the use of less powerful lasers for the same task ${ }^{18}$. Hence when maximally utilizing an SLM's addressable area, a GPC Light Shaper is useful, regardless of what light shaping technique is subsequently done with the SLM.

\subsection{Matched filtering GPC}

By borrowing features from phase-only correlation, matched filtering Generalized Phase Contrast (mGPC) combines the respective strongholds and advantages of GPC and diffractive beam shaping (e.g. holography). Similar to GPC, mGPC does not suffer from a strong un-diffracted zero-order light, ghost orders and spurious phase variations. Likewise, it is also straightforward to encode SLM phase patterns, only requiring translated copies of the same basis shape. Hence, due to the similar $4 f$ geometries, mGPC shares GPC's advantages over digital holography ${ }^{19}$. However, with the additional correlation part, mGPC also gathers light into strongly focused spikes without squeezing the field of view or working are in the sample. Instead of a PCF, mGPC uses a matched filter typically composed of concentric phase shifting rings that align to the lobes of an Airy-like function resulting from the Fourier transformed phase disks on the SLM. As the Airylike function is effectively "rectified", the planar phase at the Fourier plane results to focused beams at the output, i.e. higher peak intensities. This focusing, of course, comes at the price of losing GPC's ability of forming contiguous extended areas of light. As in image detection, the correlation part also makes mGPC tolerant to input noise such as mild phase aberrations, hence, being able to work even with consumer grade pico projectors based on liquid crystal on silicon $(\mathrm{LCoS})^{20}$. GPC, on the other hand, will also reveal the surface imperfections of a consumer projector's LCoS modulator. Hence, mGPC is attractive for lowering the cost of the cell-BOCS. A related technique to mGPC, phase-only correlation 
of amplitude patterns, have previously been used to sort particles ${ }^{21}$. Due to the $\sim 4 \mathrm{X}$ intensity gain associated with GPC, mGPC would be more effective in similar sorting experiments.

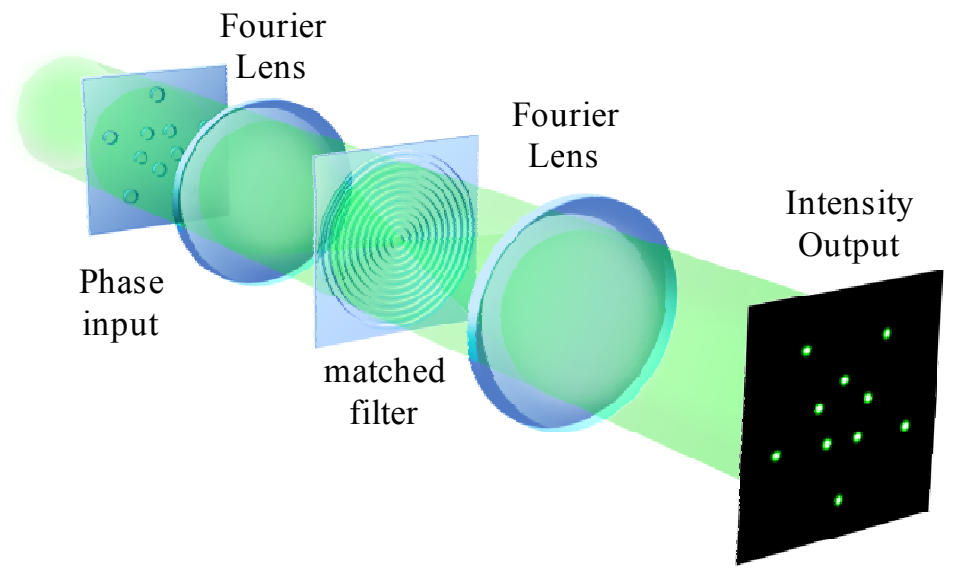

Figure 3. Setup for mGPC. Input circular phase patterns are mapped into intense output spikes by combining the operating principles of GPC and phase only correlation. The matched filter contains concentric rings that coincide with the Airy-like function's lobes.

\subsection{Binary diffraction gratings}

Despite some of the disadvantages of diffractive approaches when compared to GPC or mGPC, binary gratings that form translated focused spots at the Fourier plane has some practical advantages, making it worth considering. The setup does not require a calibrated PCF or matched filter, hence slightly lowering the bill of materials, eliminating alignment requirements for the spatial filter and allowing more freedom in the scaling of the SLM patterns. Blazed gratings are more efficient, but would however require a multi-level phase SLM. Similar to mGPC, binary gratings can also be used with cheaper consumer LCoS projectors. Unlike a complex computer generated hologram, gratings are relatively easy to calculate by mapping the output spot location to the spatial periodicity of the grating. Since cells of interest are rare, the whole SLM can be configured for a single spot and still maintain a high enough intensity to catapult the cell.

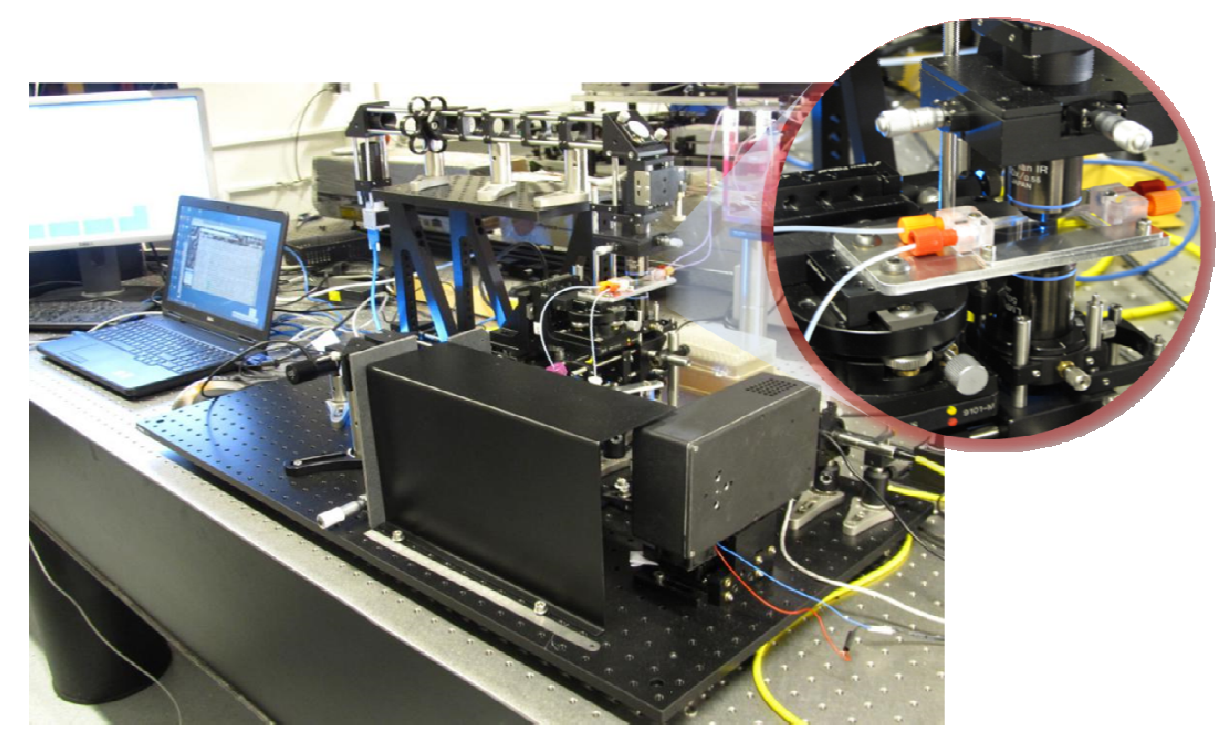

Figure 4. The cell-BOCS hardware consisting of a light shaping module, a microscope that sends images to a computer for image processing and the microfluidics system consisting of a microfluidics chip and automated syringe pumps. The inset shows a zoom in to the microfluidic chip situated close to the bottom objective that deliver the shaped laser light and the top objective that is used for image grabbing. 


\section{EXPERIMENTS}

For our initial demonstrations we used binary gratings encoded on a consumer projector. GPC or mGPC would be done after finalizing the parameters for the PCF or matched filter. Laser light $(\lambda=1070 \mathrm{~nm})$ shaped by the SLM and subsequently focused into the microfluidic chip. Targeted red blood cells are optically sorted in a plastic microfluidic chip (Epigem) and having a crossing similar to Fig. 1. Channels containing the red blood cell (RBC) samples and the destination buffer intersect at this analysis window. A CMOS camera is used to monitor all the cells passing through the window. Image processing is performed to the grabbed frames to subsequently detect RBCs that fit certain morphological parameters set by the user (Fig. 5). The SLM is updated in real time based on the location of the detected cell. The redrawn light distribution then pushes the targeted cells away from the input stream, pushing them into the collection stream. The sorted cells are later collected here for further analysis. Figure 6 shows snapshots as an RBC enters the detection window, gets detected, and then gets pushed into the top stream.

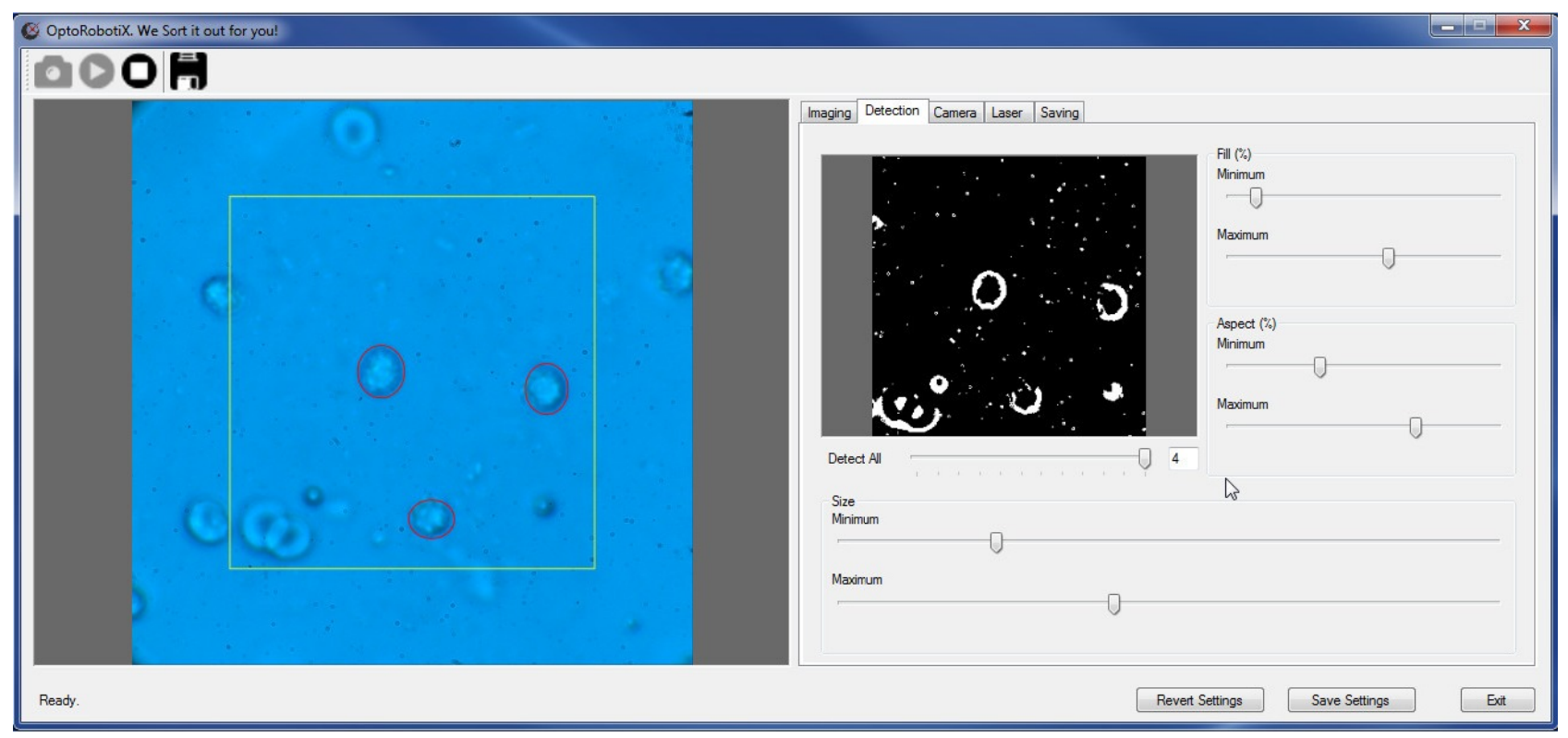

Figure 5. Software interface of the Cell-BOCS showing several cells identified against smaller particles and clumped cells in the background. A simple blob detection is currently used in the software.
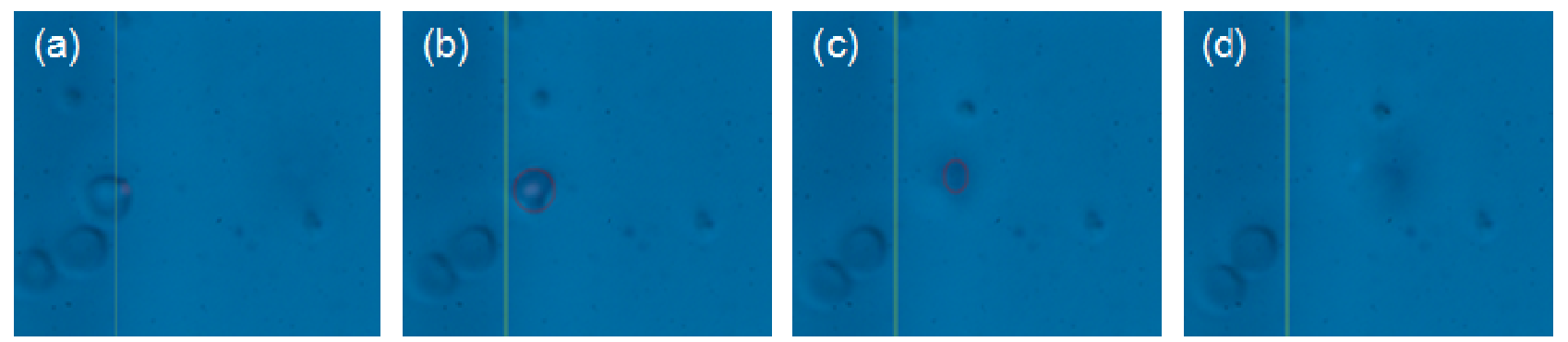

Figure 6. Detection and catapulting. A cell enters the detection region of interest (a). Overlay graphics (red ellipse) indicates that the cell is detected, and the laser is switched into the cell's location (b). As the laser-catapulted cell starts to move and become out of focus, detection continues to follow its lateral position and maintain laser catapulting (c). After enough pushing the cell becomes less visible and is eventually dragged in the upper flow (d).

\section{CONCLUSION AND OUTLOOK}

Several efficient light shaping techniques for optical cell sorting have been discussed. Practical considerations such as device cost and robustness were also taken into account. Among those considered, the least efficient light shaping technique, binary gratings, have been demonstrated due to the relative ease of its implementation. Successful sorting of 
RBCs have therefore been demonstrated at moderate flow rates that can be handled with computer image processing. We anticipate significant reductions in required input laser power when we implement GPC, mGPC or other novel phaseonly light shaping techniques.

\section{ACKNOWLEDGEMENTS}

This work has been supported by the Combined Molecular Microscopy for Therapy and Personalized Medication in Rare Anaemias Treatments (CoMMiTMent) FP7 collaborative project (Grant agreement number: 602121). We also acknowledge Epigem Ltd for lending their expertise in microfluidic device fabrication.

\section{REFERENCES}

[1] Fu, A. Y., Spence, C., Scherer, A., Arnold, F. H.., Quake, S. R., "A microfabricated fluorescence-activated cell sorter," Nat Biotech 17 (11), 1109-1111, Nature America Inc. (1999).

[2] Glückstad, J., “Sorting particles with light,” Nature Materials 3, 9-10 (2004).

[3] Sleijfer, S., Gratama, J.-W., Sieuwerts, A. M., Kraan, J., Martens, J. W. M.., Foekens, J. A., “Circulating tumour cell detection on its way to routine diagnostic implementation," Eur. J. Cancer 43 (18), 2645-2650, Elsevier (2007).

[4] Fulwyler, M. J., "Electronic separation of biological cells by volume," Science 80, 150 (3698), 910-911 (1965).

[5] MacDonald, M. P., Spalding, G. C.., Dholakia, K., "Microfluidic sorting in an optical lattice," Nature 426 (6965), 421-424 (2003).

[6] Ashkin, A., “Acceleration and Trapping of Particles by Radiation Pressure,” Phys. Rev. Lett. 24 (4), 156-159 (1970).

[7] Tanaka, Y., Kawada, H., Hirano, K., Ishikawa, M.., Kitajima, H., "Automated manipulation of non-spherical micro-objects using optical tweezers combined with image processing techniques," Opt. Express 16 (19), 1511515122 (2008).

[8] Tauro, S., Bañas, A., Palima, D.., Glückstad, J., "Dynamic axial stabilization of counter-propagating beam-traps with feedback control," Opt. Express 18 (17), 18217-18222 (2010).

[9] Bañas, A., Palima, D., Pedersen, F., Glückstad, J., "Development of a compact Bio-Optofluidic Cell Sorter," Proc. SPIE Vol. 8274, 82740N (2012).

[10] Ulriksen, H., Thogersen, J., Keiding, S., P-Nielsen, I., Dam, J., Palima, D., Stapelfeldt, H.., Glückstad, J., "Independent trapping, manipulation and characterization by an all-optical biophotonics workstation," J. Eur. Opt. Soc. Rapid Publ. 3, 08034 (2008).

[11] Glückstad, J.,, Palima, D., Generalized Phase Contrast: Applications in Optics and Photonics, Springer Series in Optical Sciences Vol. 146, 1-311 (2009).

[12] Palima, D., Glückstad, J., "Diffractive generalized phase contrast for adaptive phase imaging and optical security," Opt. Express 20 (2), 1370-1377 (2012).

[13] Palima, D., Banas, A., Villangca, M., Glückstad, J., “GPC and quantitative phase imaging,” Proc. SPIE (2016).

[14] Daria, V., Rodrigo, P., Sinzinger, S. and Glückstad, J, "Phase-only optical decryption in a planar integrated micro-optics system," Opt. Eng. 43 (10), 2223-2227 (2004).

[15] Rodrigo, P., Gammelgaard, L., Bøggild, P., P-Nielsen, I., Glückstad, J., “Actuation of microfabricated tools using multiple GPC-based counterpropagating-beam traps," Opt. Express 13 (18), 6899-6904 (2005).

[16] Bañas, A., Palima, D., Villangca, M., Aabo, T., Glückstad, J., "GPC light shaper for speckle-free one- and twophoton contiguous pattern excitation," Opt. Express 22, 23759-23769 (2014).

[17] Bañas, A., Kopylov, O., Villangca, M., Palima, D., Glückstad, J., "GPC Light Shaper: static and dynamic 
experimental demonstrations," Opt. Express 22, 5299-5311 (2014).

[18] Villangca, M., Bañas, A., Palima, D., Glückstad, J., "GPC-enhanced read-out of holograms," Opt. Commun. 351, 121-127 (2015).

[19] Palima, D., Glückstad, J., "Comparison of generalized phase contrast and computer generated holography for laser image projection,” Opt. Express 16 (8), 5338-5349 (2008).

[20] Bañas, A., Palima, D.., Glückstad, J., "Matched-filtering generalized phase contrast using LCoS pico-projectors for beam-forming," Opt. Express 20 (9), 9705-9712 (2012).

[21] Perch-Nielsen, I., Palima, D., Dam, J. S.., Glückstad, J., "Parallel particle identification and separation for active optical sorting," J. Opt. A Pure Appl. Opt. 11 (3), 034013 (2009). 